\title{
The dark side of open access
}

\author{
Ørjan G. Martinsen 1,2,3 \\ 1. Editor-in-chief, Journal of Electrical Biompedance \\ 2. Department of Physics, University of Oslo, Norway \\ 3. Department of Clinical and Biomedical Engineering, Oslo University Hospital, Norway
}

\begin{abstract}
Every day - with no exceptions - I get e-mails from editors wanting me to publish my research in their journals. Just a few years ago, I got no such e-mails at all. What happened? In my opinion, this phenomenon is unfortunately symptomatic of a new and very unfortunate trend in international research.
\end{abstract}

The first scientific journal, Philosophical Transactions, was established in 1665. Today, there are thousands of journals covering virtually every possible field of research. The journals have traditionally been run by publishing houses or large organizations and to read the journals, institutions or individuals pay a subscription fee.

The first free scientific online archive was arXiv.org. It was started in 1991 as a preprint library for physicists. Later different versions of open access journals appeared; some are offered both as printed and online journals and others as online journals only. The obvious advantage is a more fair and easy access to the scientific literature - also for those who could earlier not afford the high subscription rates. The challenge for the publishers is of course to change the economic system for scientific publishing so that their income is not reduced. Hence, the cost is typically transferred to the authors.

With a pay per published article based system, there is an inherent economic incentive for a journal to publish as many articles as possible. Many established, high-quality journals are able to balance this in a responsible and serious way. Nevertheless, this situation spurs the creativity of many profit-seeking players in this market. More than one creative soul has made the following calculation:

If I start $X$ number of journals, which each publish $Y$ number of articles each year, and I charge $Z$ euros per article, than I will earn $X \cdot Y \cdot Z$ euros per year.
Moreover, this is a very pleasant calculation to do, because if you are a bit optimistic about the numbers (and who doesn't want to be optimistic) then the product easily becomes an inspiringly high amount of money. The danger is now that this model very easily shifts the focus from quality to quantity, because the income linearly scales with the number of published articles.

A couple of years ago I started to collaborate with a researcher who was new to bioimpedance. He had found some articles in an open access journal where a very simple resistance (or impedance) device gave results that would simply revolutionize medicine if the reports were correct. It was apparent for anyone with some basic bioimpedance knowledge that the results described in these articles were simply fake. I contacted the editor-in-chief of the journal, which was published by a company responsible for a huge numbers of journals, many of them with high impact factors. The editor-in-chief replied to me that "sometimes the review process can go a bit too quickly" and he suggested that I could write a letter to the editor. I was shocked - can you believe an editor-in-chief saying "sometimes the review process can go a bit too quickly"? My opinion is that if there is one single responsibility that the editor-in-chief has, then it is that the review process does not "go too quickly"!

The world has enough journals of physics or journals of medicine. The reason for starting another journal should not primarily be economic profit and in my opinion, one should basically only consider starting a new journal if either there is a lack of good journals in the specific subject area or you want to start a platinum open access journal, i.e. an open access journal with no publication fee. I am not against publication fees, and I realize that when this journal grows beyond a certain limit, we will not be able to run it on a purely voluntary basis anymore. However, the publication fee should be kept low and other ways of getting income should be considered, like e.g. advertisements. And most important of all; the focus must never be allowed to shift from quality to quantity. 